\title{
Pustulose exanthématique aiguë généralisée
}

\author{
Kristyna Cleminson MD, Natalie Cunningham MD
}

Citation : CMAJ 2020 October 19;192:E1268. doi : 10.1503/cmaj.200324-f

Voir la version anglaise de l'article ici : www.cmaj.ca/lookup/doi/10.1503/cmaj.200324

$\mathbf{U}$ ne femme de 71 ans présentant de la bradycardie, mais autrement en santé, a été hospitalisée pour l'installation d'un stimulateur cardiaque. Elle a reçu une dose de céfazoline durant l'intervention. Le lendemain, elle présentait une éruption morbilliforme prurigineuse aux extrémités inférieures. Deux jours plus tard, l'éruption était généralisée et accompagnée de plaques érythémateuses confluentes recouvertes de pustules non folliculaires (figure 1). Les muqueuses étaient intactes. La patiente était fébrile et présentait une leucocytose $\left(34 \times 10^{9} / \mathrm{L}\right.$ [plage normale $\left.\left.4,5-11 \times 10^{9} / \mathrm{L}\right]\right)$. Un diagnostic de pustulose exanthématique aiguë généralisée attribuable à la céfazoline a été posé, appuyé par un examen histologique, qui a montré la présence de pustules sous cornées, d'une spongiose, de neutrophiles périvasculaires et d'une exocytose d'éosinophiles ${ }^{1}$. La patiente n'a reçu aucune autre dose de céfazoline et s'est vu prescrire une crème à base de bétaméthasone $(0,1 \%)$, à appliquer sur la peau affectée 2 fois par jour pendant 2 semaines. Aucun stéroïde systémique n'a été administré. L'éruption s'est complètement résorbée.

La pustulose exanthématique aiguë généralisée est une réaction d'hypersensibilité médicamenteuse de type IV grave, mais rare, associée aux antibiotiques, aux inhibiteurs des canaux calciques, aux antipaludiques, à l'acétaminophène, aux anti-inflammatoires non stéroïdiens et à la terbinafine. Elle apparaît soudainement, dans les $2-5$ jours suivant l'exposition au médicament. L'éruption est caractérisée par la présence de pustules non folliculaires sur une peau érythémateuse, généralement dans les régions intertrigineuses². Les pustules persistent quelques jours, après quoi une desquamation superficielle survient dans les régions touchées; les muqueuses restent habituellement intactes ${ }^{3}$. Les patients disent souvent ressentir des démangeaisons et une sensation de brûlure ${ }^{1,3}$. Des atteintes rénales et hépatiques ont parfois été observées ${ }^{1,2}$. Le diagnostic différentiel comprend l'infection, le psoriasis pustulaire et la nécrolyse épidermique toxique ${ }^{1,3}$.

Généralement, les symptômes se résorbent en 2 semaines. Toutefois, chez les patients fragiles qui développent de la fièvre et une surinfection, elle est associée à un taux de mortalité de $5 \%{ }^{2}$. Un test épicutané réalisé après le rétablissement a une sensibilité de $80 \%$ et une spécificité de $85 \%$ pour l'identification de l'agent en caus $\mathrm{e}^{1,2}$. Le traitement comprend l'arrêt du médicament à l'origine de la réaction, de même que l'administration d'antipyrétiques et de corticostéroïdes topiques ou systémiques pour diminuer le prurit et l'inflammation ${ }^{2,3}$.

\section{Références}

1. Speeckaert MM, Speeckaert R, Lambert J, et al. Acute generalized exanthematous pustulosis: an overview of the clinical, immunological and diagnostic concepts. Eur J Dermatol 2010;20:425-33.

2. De A, Das S, Sarda A, et al. Acute generalized exanthematous pustulosis: an update. Indian J Dermatol 2018;63:22-9.

3. Sidoroff A, Halevy S, Bavinck J, et al. Acute generalized exanthematous pustolusis (AGEP) a clinical reaction pattern. J Cutan Pathol 2020;28:113-9.

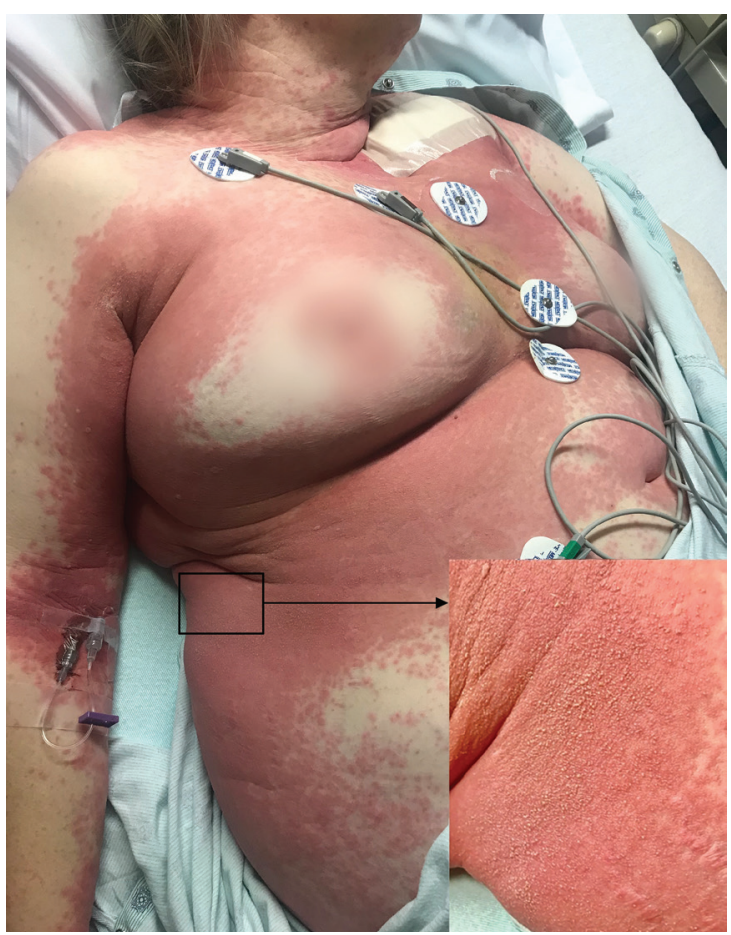

Figure 1 : Éruption érythémateuse généralisée touchant particulièrement les plis de flexion et recouverte de pustules non folliculaires chez une femme de 71 ans présentant une pustulose exanthématique aiguë généralisée.

Intérêts concurrents : Aucun déclaré.

Cet article a été révisé par des pairs.

Les auteurs ont obtenu le consentement de la patiente.

Affiliation : Division de dermatologie clinique et des sciences de la peau, Département de médecine, Université Dalhousie, Halifax (Nouvelle-Écosse)

Propriété intellectuelle du contenu : Il s'agit d'un article en libre accès distribué conformément aux modalités de la licence Creative Commons Attribution (CC BY-NC-ND 4.0), qui permet l'utilisation, la diffusion et la reproduction dans tout médium à la condition que la publication originale soit adéquatement citée, que l'utilisation se fasse à des fins non commerciales (c.-à-d., recherche ou éducation) et qu'aucune modification ni adaptation n'y soit apportée. Voir : https://creativecommons.org/licenses/by-nc-nd/4.0/.

Correspondance : Kristyna Cleminson, k.cleminson@dal.ca 